\title{
Estresse oxidativo e aumento da apoptose em neutrófilos de cães com azotemia pré-renal
}

\author{
[Oxidative stress and increase in apoptosis index of neutrophils from \\ dogs with prerenal azotemia] \\ A.C.R.A. Silva ${ }^{1}$, B.F.M. Almeida ${ }^{1}$, C.S. Soeiro ${ }^{1}$, W.L. Ferreira ${ }^{2}$, \\ V.M.F. Lima ${ }^{3}$, P.C. Ciarlini ${ }^{3}$

\footnotetext{
${ }^{1}$ Aluno de pós-graduação - Faculdade de Medicina Veterinária de Araçatuba - UNESP - Araçatuba, SP

${ }^{2}$ Faculdade de Medicina Veterinária de Araçatuba - UNESP - Araçatuba, SP
}

\begin{abstract}
RESUMO
O presente trabalho tem como objetivo testar a hipótese de que, à semelhança do que ocorre na uremia, cães com azotemia pré-renal sofrem estresse oxidativo, o qual está relacionado com alterações do metabolismo oxidativo e apoptose dos neutrófilos. Para tal, foi determinada a peroxidação lipídica pela quantificação do malondialdeído (MDA) e o status antioxidante total do plasma de 15 cães normais e 10 com azotemia pré-renal, correlacionando-os com a produção de superóxido e o índice apoptótico dos neutrófilos. As determinações do MDA e do status antioxidante total foram estabelecidas empregando-se um conjunto de reagentes comerciais. Por meio de citometria de fluxo capilar, a produção de superóxido e a apoptose de neutrófilos isolados de sangue periférico foram determinadas utilizando-se a sonda hidroetidina e o sistema anexina V-PE, respectivamente. Cães azotêmicos $(26,29 \pm 5,32 \mathrm{~g} / \mathrm{L})$ apresentaram menor concentração $(\mathrm{p}=0,0264)$ do antioxidante albumina em relação ao grupo-controle $(30,36 \pm 3,29 \mathrm{~g} / \mathrm{L})$ e também uma menor $(\mathrm{p}=0,0027)$ capacidade antioxidante total $(2,36 \pm 0,32$ versus $2,73 \pm 0,24 \mathrm{mmol} / \mathrm{L})$, enquanto não houve alteração da peroxidação lipídica plasmática e da produção de superóxido neutrofílica. Concluiu-se que, à semelhança do que ocorre na uremia, condições azotêmicas pré-renais no cão causam estresse oxidativo e aceleração da apoptose dos neutrófilos.
\end{abstract}

Palavras-chave: metabolismo oxidativo, peroxidação lipídica, capacidade antioxidante, imunossupressão

\begin{abstract}
This study aims to test the hypothesis that, similarly to what occurs in uremia, dogs with prerenal azotemia suffer oxidative stress associated with changes in oxidative metabolism and apoptosis in neutrophils. For this purpose, fifteen normal dogs and ten with prerenal azotemia had lipid peroxidation determined by quantifying the malondialdehyde (MDA) and had plasma total antioxidant status evaluated, correlating them with the superoxide production and apoptotic index of neutrophils. MDA and plasma total antioxidant status were determined using commercial reagents. Using capillary flow cytometry, superoxide production and apoptosis were determined from isolated neutrophils of peripheral blood using the hydrithidine and Annexin V-PE probe system, respectively. Azotemic dogs $(26.29 \pm 5.32 \mathrm{~g} / \mathrm{L})$ had a lower concentration $(p=0.0264)$ of the plasma antioxidant albumin than the control group $(30.36 \pm 3.29 \mathrm{~g} / \mathrm{L})$ and also had lower $(p=0.0027)$ total antioxidant status $(2.36 \pm 0.32$ versus $2.73 \pm 0.24 \mathrm{mmol} / \mathrm{L})$, while no alterations were observed in plasma lipid peroxidation and superoxide production. It was concluded that, similarly to what occurs in uremia, prerenal azotemia causes oxidative stress and acceleration of neutrophil apoptosis in dogs.
\end{abstract}

Keywords: oxidative metabolism, lipidic peroxidation, antioxidant status, immunosuppression

Recebido em 29 de agosto de 2011

Aceito em 9 de setembro de 2012

*Autor para correspondência (corresponding author)

E-mail: bfmalmeida@yahoo.com.br 


\section{INTRODUÇÃO}

Quando devidamente estimulados, os neutrófilos produzem espécies reativas de oxigênio (ERO) a partir do sistema NADPH-oxidase (Huimin et al., 2000), em que o NADPH é oxidado pela ativação da enzima NADPH-oxidase, transferindo dois elétrons para cada duas moléculas de oxigênio e, assim, gerando o ânion superóxido, essencial para função bactericida dos neutrófilos (Dobos et al., 1997). A partir do superóxido, a enzima mieloperoxidase forma peróxido de hidrogênio e radical hidroxila (Huimin et al., 2000). Embora as ERO sejam necessárias para o mecanismo de defesa dos neutrófilos, os radicais livres produzidos podem interagir em excesso e lesar diversas estruturas celulares, induzindo, assim, à peroxidação lipídica e à aceleração da apoptose (Cendoroglo et al., 1999). Os produtos da peroxidação lipídica, tal como o malondialdeído (MDA), podem ser usados como indicadores da ação dos radicais livres, e sua quantificação é um parâmetro importante para a avaliação do estresse oxidativo (Antunes et al., 2008).

A concentração de ERO e a limitação da peroxidação lipídica são mantidas por meio de defesas antioxidantes que interrompem a cadeia de peroxidação, removendo as ERO (Brown, 2008). Quando ocorre um aumento na produção de ERO e consequente diminuição da defesa antioxidante, este processo desencadeia o estresse oxidativo no qual o excesso causa danos e disfunções em muitos componentes vitais para a manutenção da célula (Vaziri et al., 2003). Neutrófilos que têm o estímulo para produção de superóxido exacerbado durante a explosão respiratória podem sofrer apoptose, uma vez que as ERO danificam os próprios componentes celulares quando a capacidade antioxidante da célula é excedida (Cendoroglo et al., 1999).

Em pacientes humanos, a infecção bacteriana é a principal causa de morte em pacientes nefropatas terminais (Cendoroglo et al., 1999). Acredita-se que este fato esteja associado à disfunção dos neutrófilos causada pelo estresse oxidativo da uremia; esta teria ação inibidora sobre a função dos neutrófilos (Chonchol, 2006). Pesquisas com neutrófilos humanos identificaram que pacientes com insuficiência renal crônica (IRC) apresentam uma proteína inibidora de granulócitos que compromete a atividade quimiotática e o metabolismo oxidativo dos neutrófilos, diminuindo a atividade glicolítica e os níveis de ATP intracelular (Hörl et al., 1990). Cendoroglo et al. (1999), ao avaliarem o efeito do plasma urêmico sobre a função e a apoptose de neutrófilos normais, observaram maiores taxas de apoptose, menor produção de superóxido e menor índice de fagocitose.

Existe escassez de informações em relação à disfunção neutrofílica em condições de azotemia em cães. Pesquisas constataram que o plasma de cães sadios, quando enriquecido com ureia, promove aceleração da apoptose (Trinconi et al., 2008) e redução do metabolismo oxidativo dos neutrófilos (Trevelin et al., 2008). A uremia em cães, à semelhança do que ocorre em humanos, também afeta o metabolismo oxidativo dos neutrófilos in vitro (Barbosa et al., 2010). Recentemente, foi comprovado que a IRC causa estresse oxidativo em gatos (Keegan e Webb, 2010) e em cães (Silva, 2011).

Os cães são comumente afetados por diversas causas de azotemia não renal, como dietas hiperproteicas, aumento do metabolismo proteico e cardiopatias. Há necessidade de se investigar se o estresse oxidativo já comprovado na insuficiência renal em humanos (Rysz et al., 2004), gatos (Keegan e Webb, 2010) e cães (Silva, 2011) igualmente ocorre em outras condições azotêmicas e se este altera a produção de superóxido e a sobrevida dos neutrófilos. Para tal, foi realizada uma investigação com o objetivo de testar in vivo a hipótese de que cães com azotemia pré-renal igualmente sofrem estresse oxidativo e que esta condição está relacionada com as alterações do metabolismo oxidativo e a apoptose dos neutrófilos.

\section{MATERIAL E MÉTODOS}

Para a seleção dos animais, todos os cães foram submetidos a um exame físico geral e a exames clínicos e laboratoriais (hemograma completo, proteína plasmática total, fibrinogênio plasmático, urinálise tipo I, albumina, ureia, creatinina, ácido úrico, colesterol, glicose e frutosamina plasmática). Conforme os critérios clínicos e laboratoriais, 15 cães com valores de ureia inferiores a $8 \mathrm{mmol} / \mathrm{L}$ foram selecionados para compor o grupo-controle (GC), e 10 cães não nefropatas e sem obstrução pós-renal, com valores de ureia entre $8-35 \mathrm{mmol} / \mathrm{L}$, foram selecionados para o grupo azotêmico (GA). Animais com histórico de tratamento recente que 
pudesse interferir na função renal ou leucocitária foram excluídos do estudo.

De cada animal foram colhidos $10 \mathrm{~mL}$ de sangue total, sendo $4 \mathrm{~mL}$ destinados para avaliação do estresse oxidativo dos neutrófilos e $3 \mathrm{~mL}$ para obtenção de plasma para análises bioquímicas, sempre sendo utilizadas agulhas hipodérmicas descartáveis $25 \times 0,8 \mathrm{~mm}$ próprias para tubos siliconizados heparinizados a vácuo (Vacutainer plus plastic Heparin, Cod. 367993, BectonDickson, New Jersey, USA). Outros $3 \mathrm{~mL}$ foram acondicionados em tubos plásticos contendo EDTA-K (Vacutainer plus plastic K2 EDTA, Cod. 367841, Becton-Dickson, New Jersey, USA) para realização do hemograma. As análises laboratoriais foram realizadas logo após a obtenção das amostras sanguíneas, e o plasma utilizado para as análises bioquímicas foi armazenado a $-20^{\circ} \mathrm{C}$.

As taxas totais de leucócitos, eritrócitos e hemoglobina foram obtidas com auxílio de contador eletrônico de células sanguíneas (Contador eletrônico hematológico veterinário, Mod. CC-530, CELM, São Paulo-SP), e o volume globular pelo método microcapilar de Strumia (centrifugação 12700 G/5 minutos). A contagem diferencial de leucócitos foi realizada em esfregaços sanguíneos corados com corante hematológico panótico rápido comercial (InstantProv, NEWPROV, Pinhais- PR).

O exame de urina tipo I foi realizado utilizandose tiras reagentes comerciais (Combur ${ }^{10}$ Test $^{\circledR}$ UX, Roche). As análises bioquímicas foram realizadas em analisador bioquímico automatizado (Analisador automático BTS, mod. 370 plus, BioSystems, Barcelona, Espain). Utilizando-se conjunto de reativos comerciais, a concentração plasmática de ureia foi determinada pelo método enzimático UV (urease/glutamato desidrogenase - Urea/BUN-UV,Cod. 11516, BioSystems, Barcelona, Espain); a de creatinina pelo método cinético (picrato alcalino Creatinine, Cod.11502, BioSystems, Barcelona, Espain); a de albumina pelo método do verde de bromocresol (Albumine, Cod.11574, BioSystems, Barcelona, Espain); a de ácido úrico pelo método uricase/peroxidase (Uric acid, Cod.11802, BioSystems, Barcelona, Espain); a do colesterol pelo método enzimático oxidase/peroxidase (Cholesterol, Cód.11505, BioSystems, Barcelona, Espain); a de glicose pelo método enzimático (glicose oxidase/peroxidase - Glucosa, Cod.11503, BioSystems, Barcelona, Espain) e a de frutosamina pelo método colorimétrico de redução do NBT (Frutosamine NBT, Cod. 11046, BioSystems, Barcelona, Espain). Todas as reações bioquímicas foram processadas a $37^{\circ} \mathrm{C}$ conforme orientações dos fabricantes.

Para o isolamento dos neutrófilos, $4 \mathrm{~mL}$ e sangue total heparinizado $(10 \mathrm{UI} / \mathrm{mL})$, obtidos em frasco estéril (Labor import, Cod. 50091, Wei Hai, China), foram transferidos para tubo cônico de polipropileno estéril contendo duplo gradiente de separação composto de volumes iguais $(3 \mathrm{~mL})$ de Histopaque-1119 (Histopaque $®-1119, \quad$ Cod. 1119-1, Sigma, St. Louis, USA) e 1077 (Histopaque ${ }^{\circledR}-1077$, Cod. 1077-1, Sigma. St. Louis, USA). Após centrifugação a $340 \mathrm{G}$ por 30 minutos, a camada de polimorfonucleares foi aspirada e lavada duas vezes com solução lisante à base de cloreto de amônio $\left(4,01 \mathrm{~g} \mathrm{NH}_{4} \mathrm{Cl} ; 0,8 \mathrm{~g}\right.$ EDTA-Na; 0,42g $\mathrm{Na}_{2} \mathrm{CO}_{3}$; q.s.p. $500 \mathrm{~mL} \mathrm{H}_{2} \mathrm{O}$ ) para total lise dos eritrócitos residuais. Em seguida, a amostra foi centrifugada (100 G) durante cinco minutos com HBSS (Hanks balanced salt solution (HBSS) modified, Cod. H 9394, Sigma, St. Louis, USA) sem $\mathrm{Ca}^{2+} \mathrm{e} \mathrm{Mg}^{2+}$, e ao sedimento celular foi acrescido $1 \mathrm{~mL}$ e RPMI (Medium RPMI 1640, Cat. R0883, SigmaAldrich, St. Louis, USA). Após determinar a concentração celular em hemocitômetro e estimar a viabilidade celular pelo método de exclusão do azul tripan, a amostra foi diluída em meio RPMI para obter concentração celular final de $1 \times 10^{6} / \mathrm{mL}$, com pureza e viabilidade mínimas de $90 \%$.

A peroxidação lipídica plasmática foi determinada pela quantificação de MDA, por meio do método de TBARS (substâncias reativas ao ácido tiobarbitúrico), utilizando-se um conjunto de reagente comercial (TBARS Assay Kit, cat. 10009055, Cayman Chemical Company, USA). Segundo as recomendações do fabricante, a partir de uma solução padrão comercial de MDA $(500 \mu \mathrm{M})$ e empregando-se programa computacional (GraphPad Prism, version 4, GraphPad Software Inc, San Diego, USA), foi elaborada uma curva referente às concentrações finais de $0 ; 0,625 ; 1,25 ; 2,5 ; 5 ; 10 ; 25 \mathrm{e}$ $50 \mathrm{nmol} / \mathrm{mL}$ de MDA. Cada ponto da curva foi obtido a partir do valor médio de 10 repetições. 
O status antioxidante total foi determinado pelo método de inibição de formação de cátion de ABTS $^{\circledR}$ (2.2'-Azino diethyl-bezothiazoline sulfonic acid - Total antioxidante status, cat. NX2332, Randox laboratories, UK), monitorado com padrão antioxidante específico para automatização (Anti-oxidants standard, cat. NX2615, Randox laboratories, UK).

Para a determinação de superóxido dos neutrófilos, utilizou-se a sonda hidroetidina (HE) (Hydroethidine $^{\mathrm{TM}}$, dihydroethidium bromide, Cat. 17084, Polysciences, Warrington, USA). Resumidamente, em $180 \mu \mathrm{L}$ de suspensão de neutrófilos $\left(1 \times 10^{6} / \mathrm{mL}\right)$ em RPMI, foi acrescida uma solução tamponada de $\mathrm{HE}(0,1 \mathrm{mmol} / \mathrm{L})$, sem e com adição de $20 \mu \mathrm{L}$ de PMA (Phorbol 12myristate 13-acetate (PMA), Cat. P8139, Sigma, St. Louis, USA) $(3,2 \mu \mathrm{mol} / \mathrm{mL})$. Após incubação a $37^{\circ} \mathrm{C}$ por 15 minutos, a amostra foi mantida em banho de gelo e protegida da ação luz até o momento da leitura. A concentração de superóxido foi quantificada em citômetro de fluxo capilar (Guava EasyCyte Mini ${ }^{\circledR}$, Guava Technologies, Industrial Boulevard Hayward, USA), ajustado para o comprimento de onda máximo para emissão $(593 \mathrm{~nm})$ e excitação (473nm) do brometo de etídio. Os resultados de 10.000 eventos foram analisados em programa computacional específico (Guava Express, CytoSoft Data Acquisition and Analysis Software. Personal Cell Analysis, v.4.1, 2006. Guava Technologies, Industrial Boulevard Hayward, USA). Os granulócitos foram distinguidos de debris celulares (plaquetas e eritrócitos residuais), monócitos e linfócitos contaminantes devido ao seu maior FALS (Forward-angle light scatter) e 90LS (Rigthangle light scatter). Selecionou-se a população com maior FALS típica de neutrófilos e foi considerada a unidade arbitrária da fluorescência vermelha média emitida pelo brometo de etídio formado a partir da oxidação espontânea e estimulada do HE pelo superóxido.

A taxa de apoptose foi determinada por citometria de fluxo capilar, utilizando-se sistema Anexina V-PE (Guava Nexin Kit, Cat. 45000010, Guava Technologies, USA). Para tal, a apoptose de cada cão foi realizada em duas provas. Na prova induzida, às alíquotas de $100 \mu \mathrm{L}$ de uma suspensão de neutrófilos $\left(1 \times 10^{6} / \mathrm{mL}\right)$, foram acrescidos $100 \mu \mathrm{L}$ do indutor camptotecina (Camptothecin, Cod. C9911. Sigma, St. Louis, USA) $(19,8 \mathrm{mmol} / \mathrm{L})$, enquanto na prova não induzida foi acrescido o mesmo volume de RPMI. Posteriormente, as amostras foram incubadas por uma hora a $37^{\circ} \mathrm{C}$, sendo agitadas por um minuto (600rpm) a cada 15 minutos em agitador térmico computadorizado (Thermomixer, Eppendorf, Mod. Comfort, Hamburg, Germany). Em seguida, a suspensão de células foi aliquotada em $100 \mu \mathrm{L}$, acrescida de $100 \mu \mathrm{L}$ de anexina V-PE e incubada por 20 minutos em temperatura ambiente protegida da luz. Utilizando-se citômetro de fluxo capilar, os resultados de 10.000 eventos foram analisados em programa computacional específico, sendo que, após a compensação do citômetro para o fluorocromo vermelho para reação positiva de 7AAD e fluorocromo amarelo para reação positiva da anexina $\mathrm{V}$, foi possível quantificar três populações celulares: células não apoptóticas viáveis (anexina $\mathrm{V}$ - e 7-AAD -); apoptose inicial (anexina $\mathrm{V}+\mathrm{e}$ 7-AAD-) e apoptose final ou necrose (anexina $\mathrm{V}+\mathrm{e} 7-\mathrm{AAD}+$ ).

Após os estudos das distribuições das variáveis quanto à normalidade e homocedasticidade, utilizou-se $\mathrm{o}$ teste de $\mathrm{t}$ não pareado para comparar os grupos e o coeficiente de Pearson para a correlação das variáveis paramétricas. As análises estatísticas supracitadas foram feitas com auxílio de um programa computacional estatístico (SAS/STA Software, Statistical Analysis System Institute, 1997, USA).

\section{RESULTADOS E DISCUSSÃO}

Os valores do perfil bioquímico dos cães sadios permaneceram dentro da faixa de normalidade da espécie (Tab. 1), compatível com a higidez dos animais do grupo-controle. Nos dois grupos experimentais, o exame físico-químico e de sedimento da urina não revelou qualquer alteração compatível com doença renal ou pósrenal. As concentrações de creatinina e ureia plasmática do grupo azotêmico foram significativamente maiores (Tab. 1) e dentro da faixa de aumento esperada para cães com azotemia pré-renal considerada por Kerr (2003). 
Tabela 1. Valores médios e desvios-padrão de constituintes plasmáticos de cães normais e azotêmicos, Araçatuba, São Paulo, Brasil

\begin{tabular}{lccc}
\hline & Grupo-controle & Grupo azotêmico & p-value \\
\hline Creatinina (mg/dL) & $1,06 \pm 0,10 \mathrm{a}$ & $1,61 \pm 0,74 \mathrm{~b}$ & 0,0002 \\
Ureia $(\mathrm{mg} / \mathrm{dL})$ & $46,45 \pm 9,85 \mathrm{a}$ & $66,59 \pm 17 \mathrm{~b}$ & 0,0010 \\
Glicose $(\mathrm{mg} / \mathrm{dL})$ & $75,1 \pm 10,67 \mathrm{a}$ & $106 \pm 52,29 \mathrm{~b}$ & 0,0349 \\
Frutosamina $(\mathrm{mmol} / \mathrm{L})$ & $1,95 \pm 0,44 \mathrm{a}$ & $1,72 \pm 0,15 \mathrm{a}$ & 0,1212 \\
Albumina $(\mathrm{g} / \mathrm{L})$ & $30,36 \pm 3,29 \mathrm{a}$ & $26,29 \pm 5,32 \mathrm{~b}$ & 0,0264 \\
Ácido úrico (mg/dL) & $1,33 \pm 0,59 \mathrm{a}$ & $1,27 \pm 0,72 \mathrm{a}$ & 0,8074 \\
Colesterol $(\mathrm{mg} / \mathrm{dL})$ & $161,0 \pm 104,74 \mathrm{a}$ & $117,55 \pm 91,71 \mathrm{a}$ & 0,2975 \\
MDA $(\mathrm{nmol} / \mathrm{mL})$ & $43,4 \pm 8,39 \mathrm{a}$ & $41,50 \pm 23,26 \mathrm{a}$ & 0,77 \\
Status antioxidante total $(\mathrm{mmol} / \mathrm{L})$ & $2,73 \pm 0,24 \mathrm{a}$ & $2,36 \pm 0,32 \mathrm{~b}$ & 0,0027 \\
\hline
\end{tabular}

Os maiores valores de glicose em cães com azotemia (Tab. 1) provavelmente ocorreram devido ao estresse de alguns indivíduos desse grupo que também apresentaram leucograma de estresse (dados não apresentados). Em condição de estresse, o aumento da glicemia é transitório e discreto, portanto não promove aumento de frutosamina, como observado no presente estudo. Pacientes humanos com IRC comumente apresentam hiperglicemia e elevação da frutosamina plasmática devido a uma resistência à insulina (Bilbrey et al., 1974), e estas alterações têm sido associadas ao aumento da apoptose dos neutrófilos (Tennenberg et al.,
1999). Estudos realizados revelaram que, in vitro, a hiperglicemia aumenta a apoptose dos neutrófilos de cães (Nogueira et al., 2010). Entretanto, em condições de IRC nesta espécie, a hiperglicemia não foi observada (Silva, 2011).

Os cães azotêmicos apresentaram significativa diminuição da albumina plasmática (Tab. 1). A albumina é um importante antioxidante endógeno (Araújo et al., 2006) e sua diminuição pode ter contribuído para a menor capacidade antioxidante total do plasma observada nos cães azotêmicos (Tab. 1).

Tabela 2. Metabolismo oxidativo, viabilidade e apoptose de neutrófilos de cães sadios e com azotemia pré-renal, com e sem ativação, expressa em valores médios e desvios-padrão, Araçatuba, São Paulo, Brasil

\begin{tabular}{lcc}
\hline \multirow{2}{*}{ Grupo } & \multicolumn{2}{c}{ Fluorescência média do brometo de etídio } \\
\cline { 2 - 3 } \multicolumn{1}{c}{ Controle } & Sem PMA & Com PMA \\
Azotêmico & $162,93 \pm 173,09 \mathrm{a}$ & $1182,5 \pm 1253,5 \mathrm{a}$ \\
p-value & $278,24 \pm 338,4 \mathrm{a}$ & $487,13 \pm 503,89 \mathrm{a}$ \\
\multicolumn{1}{c}{ Grupo } & 0,2722 & 0,1110 \\
\cline { 2 - 3 } \multicolumn{1}{c}{ Controle } & Sem CAM & Com CAM \\
Azotêmico & $99,74 \pm 0,20 \mathrm{a}$ & $76,02 \pm 13,43 \mathrm{a}$ \\
p-value & $98,97 \pm 0,92 \mathrm{~b}$ & $56,06 \pm 17,61 \mathrm{~b}$ \\
\hline \multicolumn{1}{c}{ Grupo } & 0,0043 & 0,0038 \\
\hline Controle & \multicolumn{3}{|}{ Com CAM } \\
Azotêmico & Sem CAM & $4,34 \pm 3,93 \mathrm{a}$ \\
p-value & $0,018 \pm 0,016 \mathrm{a}$ & $22,34 \pm 19,7 \mathrm{~b}$ \\
\hline
\end{tabular}

PMA (13-acetato de forbol éster 12-miristato); CAM (camptotecina). 
Comparado ao estresse oxidativo que ocorre na IRC descrito em humanos (Bianchi et al., 2009), gatos (Keegan e Webb, 2010) e cães (Silva, 2011), a azotemia pré-renal parece afetar a capacidade antioxidante do cão de forma mais discreta. No presente estudo, o estresse oxidativo dos cães azotêmicos caracterizou-se pela diminuição dos antioxidantes plasmáticos, sem aumento da peroxidação lipídica plasmática (Tab. 1) ou alteração significativa da produção neutrofílica de superóxido (Tab. 2). Silva (2011) também não observou em cães com IRC aumento da peroxidação lipídica plasmática (MDA), porém constatou nesta condição maior diminuição da capacidade antioxidante plasmática $(2,01 \pm 0,45 \mathrm{mmol} / \mathrm{L}) \mathrm{e}$, diferentemente disso, observou menor produção neutrofílica de superóxido.

Em pacientes humanos com IRC, os resultados sobre o metabolismo oxidativo dos neutrófilos são conflitantes. Alguns trabalhos observaram diminuição da produção de superóxido e outras espécies reativas de oxigênio (Cendoroglo et al., 1999; Sardenberg et al., 2006), enquanto outros observaram aumento dessas substâncias (McLeish et al., 1996; Rysz et al., 2004) e outros ainda não observaram quaisquer alterações no metabolismo oxidativo neutrofílico (Gastaldello et al., 2000; Anding et al., 2003). Sabe-se que parte dessas divergências são devido aos métodos de isolamento dos neutrófilos e quantificação das ERO (Sardenberg et al., 2006). A citometria de fluxo utilizando sondas é considerada uma metodologia mais sensível por permitir mensurar o metabolismo oxidativo de milhares de neutrófilos em um período muito curto de tempo, refletindo o comportamento celular com maior precisão (Zielonka e Kalyanaraman, 2010).

É provável que, além de elevadas concentrações de compostos nitrogenados, outras toxinas e proteínas que inibem o metabolismo oxidativo dos neutrófilos em condições urêmicas (Vanholder et al., 2008) não estejam presentes no plasma de cães com azotemia pré- renal.

A mensuração da apoptose dos neutrófilos por citometria de fluxo utilizando o sistema anexina V-PE permitiu uma boa avaliação dos tratamentos, quantificando e diferenciando simultaneamente a porcentagem de células viáveis e de células em apoptose final (Tab. 2). Os índices de apoptose final no teste sem indução em cães sadios e azotêmicos, em média, foi, respectivamente, 25 e 60 vezes menor do que nas amostras induzidas, demonstrando que a CAM é um bom indutor de apoptose de neutrófilos caninos (Tab. 2). Este efeito indutor de apoptose da CAM já havia sido descrito em neutrófilos de humanos (Nagami et al., 2002), porém não na espécie canina.

À semelhança do que ocorre na IRC em humanos (Sardenberg et al., 2006) e cães (Silva, 2011), na azotemia pré-renal a taxa de neutrófilos viáveis foi menor devido a um aumento significativo da taxa de apoptose espontânea e induzida com CAM (Tab. 2). Existem mais de 100 toxinas urêmicas que podem contribuir para alteração do metabolismo oxidativo e apoptose dos neutrófilos (Sardenberg et al., 2006); o p-cresol e diferentes componentes guanidínicos sabidamente afetam a função dos neutrófilos (Hirayama et al., 2000). No presente estudo, a apoptose em cães azotêmicos correlacionou-se como status antioxidante total $(\mathrm{r}=0,98)$, MDA $(\mathrm{r}=0,89)$, ácido úrico $(\mathrm{r}=0,99)$, ureia $(\mathrm{r}=0,97) \mathrm{e}$ creatinina $(\mathrm{r}=0,95)$. Estudos em humanos (Vanholder et al., 2008) e em cães observaram que in vitro as alterações do metabolismo oxidativo dos neutrófilos e sua apoptose ocorrem durante a uremia, independentemente da concentração de ureia e creatinina (Trinconi et al., 2008; Trevelin et al., 2008).

Recentemente, Barbosa et al. (2010) e Soeiro (2010) obtiveram evidências in vitro de que as toxinas urêmicas de cães promovem uma ativação inicial do metabolismo oxidativo dos neutrófilos que, posteriormente, induzem à aceleração da apoptose e à consequente diminuição da produção de superóxido. Considerando-se que a maior correlação com a apoptose em cães azotêmicos foi com o status antioxidante total, não tendo sido observada alteração na produção de superóxido, é provável que, nesta condição in vivo, a menor capacidade antioxidante do plasma seja o principal responsável pelo aumento da apoptose dos neutrófilos. 


\section{CONCLUSÃO}

Os resultados obtidos no presente estudo abrem a perspectiva de que cães portadores de azotemia pré-renal podem ter sua imunidade inespecífica comprometida e, à semelhança do que ocorre na uremia, apresentam estresse oxidativo e aceleração da apoptose dos neutrófilos.

\section{AGRADECIMENTOS}

À Fundação de Amparo à Pesquisa do Estado de São Paulo, pelo apoio financeiro, e à Laine Margareth Gabas, pelo auxílio nas análises laboratoriais.

\section{REFERÊNCIAS}

ANDING, K.; GROSS, P.; ROST, J.M. et al. The influence of uraemia and haemodialysis on neutrophil phagocytosis and antimicrobial killing. Nephrol. Dialysis Transplant., v.18, p.2067-2073, 2003.

ANTUNES, M.V.; LAZZARETTI, C.; GAMARO, G.D. et al. Estudo pré-analítico e de avaliação para determinação do malondialdeído em plasma humano por cromatografia líquida de alta eficiência, após derivatização com 2,4-dinitrofenilhidrazina. Rev. Bras. Cienc. Farmaceut., v.44, p.279-287, 2008.

ARAÚJO, M.B.; PRADA, F.J.A.; MELLO, M.A.R. Estresse oxidativo no exercício, modelos animais e intensidade do esforço. Motriz, Rev. Edu. Física, v.12, p.307-312, 2006.

BARBOSA, T.S.; MORI, C.K.; CIARLINI, P.C. Efeito inibidor do soro urêmico sobre o metabolismo oxidativo dos neutrófilos de cães. Arq. Bras. Med. Vet. Zootec., v.62, p.1352-1358, 2010.

BIANCHI, P.D.; BARP, J.; THOMÉ, F.S. et al. Efeito de uma sessão de hemodiálise sobre o estresse oxidativo sistêmico de pacientes renais crônicos terminais. J. Bras. Nefrol., v.31, p.175182,2009

BILBREY, G.; FALOONA, G.R.; WHITE, M.G. et al. Hyperglucagonemia of renal failure. J. Clin. Investigat., v.53, p.841-847, 1974.

BROWN, S.A. Oxidative stress and chronic kidney disease. Vet. Clin. North Am. Small Anim. Pract., v.38, p.157-166, 2008.
CENDOROGLO, M.; BERTRAND, L.J.; BALAKRISHNAN, V.S. et al. Neutrophil apoptosis and dysfunction in uremia. J. Am. Soc. Nephrol., v.10, p.93-100, 1999.

CHONCHOL, M. Neutrophil dysfunction and infection risk in end-stage renal disease. Seminars in Dialysis, v.19, p.291-296. 2006.

DOBOS, G.J.; BURGER, M.; KUHLMANN, J. et al. Improved cytosolic free calcium mobilization and superoxide production in bicarbonate-based peritoneal dialysis solution. Nephrol. Dialysis Transplant., v.12, p.973-977, 1997.

GASTALDELLO, K.; HUSSON, C.; WENS, R. et al. Role of complement and platelet-activating factor in the stimulation of phagocytosis and reactive oxygen species production during haemodialysis. Nephrol. Dial. Transplant., v.15, p.1638-46, 2000.

HIRAYAMA, A.; NORONHA-DUTRA, A.A.; GORDGE, M.P. et al. Inhibition of neutrophil superoxide production by uremic concentrations of guanidino compounds. J. Am. Soc. Nephrol., v.11, p.648-689, 2000 .

HÖRL, W.H.; HAAG-WEBER, M.; GEORGOPOULOS, A. et al. Physicochemical characterization of a polypeptide present in uremic serum that inhibits the biological activity of polymorphonuclear cells. Med. Sci., v.87, p.6353-6357, 1990.

HUIMIN, J.; QINJUN, X.; PEIJUN, Z. et al. Impaired GP-91 ${ }^{\text {PHOX }}$ gene expression and dysfunction of peripheral blood neutrophils in patients maintaining hemodialysis. Chinese Med. J., v.113, p.120-123, 2000.

KEEGAN, R.F.; WEBB, C.B. Oxidative stress and neutrophil function in cats with chronic renal failure. J. Vet. Inter. Med., v.24, p.514-519, 2010.

KERR, M.G. Exames laboratoriais em medicina veterinária: bioquímica clínica e hematologia veterinária. 2. ed. Roca: São Paulo, 2003. 436p.

MCLEISH, K.R.; KLEIN, J.B.; LENDERER, E.L. et al. Azotemia, TNF alpha, and LPS prime the human neutrophil oxidative burst by distinct mechanisms. Kidney Inter., v.50, p.407-416, 1996. 
NAGAMI, K.; KAWASHIMA, Y.; KUNO, H. et al. In vitro cytotoxicity assay to screen compounds for apoptosis-inducing potential on lymphocytes and neutrophils. The J. Toxicolog. Sci., v.27, p.191-203, 2002.

NOGUEIRA, M.C.R.; MORI, C.K.; BARBOSA, T.S. et al. Efeito do plasma rico em glicose sobre a apoptose dos neutrófilos de cães. Vet. Zootec., v.17, supl., p.72, 2010.

RYSZ, J.; KASIELSKI, M.; APANASIEWICZ, $\mathrm{J}$. et al. Increased hydrogen peroxide in the exhaled breath of uraemic patients unaffected by haemolialysis. Nephrol. Dial. Transplasnt., v.19, p.158-163, 2004.

SARDENBERG, C.; SUASSUNA, P.; ANDREOLI, M.C.C. et al. Effects of uraemia and dialysis modality on polymorphonuclear cell apoptosis and function. Nephrol. Dial. Transplant., v.21, p.160-165, 2006.

SAS Institute Inc. The SAS System, release 9.2. SAS Institute Inc., Cary:NC, 2008.

SILVA, A.C.R.A. Estresse oxidativo, produção de superóxido e a apoptose de neutrófilos de cães urêmicos. 2011. 54f. Dissertação (Mestrado em Ciência Animal) - Universidade Estadual Paulista, Araçatuba.

SOEIRO, C.S. Mensuração do superóxido e apoptose neutrofílica em cães azotêmicos e urêmicos. 2010. 52f. Dissertação (Mestrado em Ciência Animal) - Universidade Estadual Paulista, Araçatuba.
TENNENBERG, S.D.; FINKENAUER, R.; DWIVEDI, A. Absence of lipopolysaccharideinduced inhibition of neutrophil apoptosis in patients with diabetes. Arch. Surg., v.134, p.1229-1234, 1999.

TREVELIN, S.C.; TRINCONI, C.M.; BARBOSA, T.S. et al. Efeito do plasma rico em ureia sobre o metabolismo oxidativo dos neutrófilos de cães. Vet. Zootec., v.15, supl., p.97, 2008.

TRINCONI, C.M.; TREVELIN, S.C.; BARBOSA, T.S. et al. Efeito do plasma rico em ureia sobre a apoptose de neutrófilos de cães. Vet. Zootec., v.15, supl., p.96, 2008.

VANHOLDER, R.; VAN LAECKE, S.; GLORIEUX, G. What is new in uremic toxicity? Pediatr. Nephrol., v.23, p.1211-1221, 2008.

VAZIRI, N.D.; DICUS, M.; HO, N.D. et al. Oxidative stress and dysregulation of superoxide dismutase and NADPH oxidase in renal insufficiency. Kidney Int., v.63, p.179-185, 2003.

ZIELONKA， J.; KALYANARAMAN, B. Hydroethidine- and MitoSOX-derived red fluorescence is not a reliable indicator of intracellular superoxide formation: Another inconvenient truth. Free Rad. Biol. Med., v.48, p.983-1001, 2010. 